\title{
Slingshot-1L, a cofilin phosphatase, induces primary breast cancer metastasis
}

\author{
Chen Chen ${ }^{1, *}$, Yusufu Maimaiti ${ }^{2, *}$, Shen Zhijun ${ }^{3}$, Liu Zeming1, Guo Yawen ${ }^{1}$, Yu Pan ${ }^{1}$ \\ and Huang $\mathrm{TaO}^{1}$ \\ ${ }^{1}$ Department of Breast and Thyroid Surgery, Union Hospital, Tongji Medical College, Huazhong University of Science and \\ Technology, Wuhan 430022, P.R. China \\ ${ }^{2}$ Department of General Surgery (Research Institute of Minimally Invasive), People's Hospital of Xinjiang Uygur Autonomous \\ Region, Urumqi 830000, P.R. China \\ ${ }^{3}$ Clinical Laboratory, The Third People's Hospital of Hubei Province, Wuhan 430000, P.R. China \\ *These authors have contributed equally to this work \\ Corresponding to: Huang Tao, email: huangtaowh@163.com
}

Keywords: breast cancer, cofilin, slingshot, membrane protrusionm, actin dynamics

Received: May 10, $2017 \quad$ Accepted: June 28, $2017 \quad$ Published: August 03, 2017

Copyright: Chen et al. This is an open-access article distributed under the terms of the Creative Commons Attribution License 3.0 (CC BY 3.0), which permits unrestricted use, distribution, and reproduction in any medium, provided the original author and source are credited.

\section{ABSTRACT}

\begin{abstract}
Slingshot (SSH) is a member of the conserved family of cofilin phosphatases that plays a critical role in cell membrane protrusion and migration by transforming inactive phosphorylated cofilin to an active form. SSH-like protein 1 (SSH-1L) expression is detected in various types of tumors; insulin induces the phosphatases activity of SSH$1 \mathrm{~L}$ in a phosphoinositide 3-kinase-dependent manner. However, little is known about the expression and role of SSH-1L in breast cancer. Here, we analyzed 295 human breast cancer tissue specimens for SSH-1L expression by immunohistochemistry. The correlation between SSH-1L level and patients' clinical characteristics was analyzed with Pearson's $\chi^{2}$ test. The function of SSH-1L was evaluated by gene knockdown and quantitative real-time polymerase chain reaction detection of cofilin expression in MDA-MB-231, MCF-7, and SK-BR-3 human breast cancer cell lines. SSH$1 \mathrm{~L}$ expression was detected in $\mathbf{8 8 . 1} \%$ of tissue specimens by immunohistochemistry and was strongly associated with increased metastasis and mortality. Loss of SSH-1L expression decreased the nonphosphorylated, active form of cofilin in SK-BR-3 and MDA-MB-231 cell lines, which was associated with reduced cell motility. Accordingly, SSH-1L/cofilin signaling played a critical role in primary breast cancer metastasis and was a potential therapeutic target for breast cancer treatment.
\end{abstract}

\section{INTRODUCTION}

Cell migration plays a major role in tumor invasion and metastasis; it is initiated by membrane protrusion, which is mediated by the dynamics of actin filaments [1-2] that assemble as a network at the leading edge of migrating cells [3]. Cofilin stimulates actin depolymerization and severance of actin filaments at the protruding ends, thereby promoting their rapid turnover and determining the direction of cell movement [4-5]. Cofilin activity is regulated by its phosphorylation status, and is negatively regulated by serine/threonine kinases, LIM kinases, and testis-specific protein kinase via phosphorylation at Ser-3 [6-8]. Phospho-cofilin is dephosphorylated and reactivated by Slingshot (SSH), a member of the conserved family of protein phosphatases [9-10].

In mammalian cells, SSH phosphatases are encoded by three genes (SSH-1, -2, and -3). SSH-like proteins 1, 2, and 3 (SSH-1L, SSH-2L, and SSH-3L, respectively) exhibit distinct tissue expression patterns, subcellular distribution, and activities [9, 11]. SSH-1L expression fluctuates over the course of the cell cycle in vitro [12]. Insulin-stimulated MCF-7 cells exhibit increased SSH-1L activity and cofilin dephosphorylation, which is abrogated by phosphoinositide 
3-kinase (PI3K) inhibition [13-15]; moreover, in these cells $\mathrm{SSH}-1 \mathrm{~L}$ accumulates in protrusions where active cofilin is concentrated and directly binds insulin receptor substrate- 4 [16]. The interaction of SSH-1L with F-actin determines its activation and is required for the chemotactic response of cells $[6,8]$. The mitotic kinase Aurora (Aur)-A, which induces mammary cell migration, induces SSH-1L expression in breast cancer [17], implying that the regulation of cell migration by Aurora-A may be achieved by modulation of SSH-1L expression.

Breast cancer is one of the most common malignancies worldwide with poor prognosis [18-19]. Breast cancer metastasis has been extensively [20-21] and accumulating evidence implicates cofilin signaling as a major determinant of this process [22-23]. However, there is still relatively little information on the role of the cofilin regulatory factor $\mathrm{SSH}-1 \mathrm{~L}$ in breast cancer.

We addressed this in the present study by investigating SSH-1L expression in human breast cancer tissue and its correlation with clinical features such as metastasis and mortality. We also examined the effect of SSH-1L knockdown on cofilin phosphorylation and breast cancer cell motility and the underlying mechanisms. The results indicate that SSH-1L stimulates breast cancer cell migration via dephosphorylation of cofilin, thereby promoting metastasis. Thus, targeting SSH-1L is a potential therapeutic strategy for preventing breast cancer progression.

\section{RESULTS}

\section{SSH-1L expression in human breast cancer tissue is correlated with lymph node metastasis and poor prognosis}

A tissue microarray containing 295 human breast cancer patient specimens was used for immunohistochemical analysis. SSH-1L was detected in 260/295 samples. We established a standard protocol to define the intensity of cytoplasmic labeling, with each sample was graded on a scale of 0-3 (Figure 1A). We also investigated the correlation between SSH-1L expression and overall survival of patients. We found that patients who were negative for SSH-1L expression had higher survival rates than those who were positive (Figure 1B). In addition, SSH-1L expression, tumor stage, node stage, and TNM stage influenced the overall survival according to the log-rank test (Table 1). In the multivariate Cox regression analysis, $\mathrm{SSH}-1 \mathrm{~L}$ expression was significantly associated with patient prognosis $(\mathrm{P}=0.038$, 95\% confidence interval: 1.052-5.633) (Table 2 ). In addition, lymph node metastasis rate was higher in patients who were positive for SSH-1L expression than in those who were negative $(\mathrm{P}=0.017$; Table 3$)$.

\section{SSH-1L expression is associated with cofilin activity in breast cancer cells}

Cofilin is inactivated by phosphorylation at Ser-3 by LIM kinase and reactivated by dephosphorylation by SSH-1L [13]. This determines the invasive and metastatic phenotype of tumor cells [4, 22]; moreover, our previous work demonstrated that cofilin overexpression is associated with thyroid cancer cell migration [24]. In this study, we examined the role of SSH-1L on breast cancer cell migration and found that SSH-1L was up-regulated in breast cancer cell lines with high metastatic potential. In addition, siRNA-mediated knockdown of SSH-1L in SK-BR-3 and MDA-MB-231 cells increased cofilin phosphorylation in both cell lines (Figure 2B, 2C). These results suggest that SSH-1L expression is associated with cofilin activity in breast cancer.

\section{Loss of SSH-1L expression decreases breast cancer cell migration}

The effect of SSH-1L knockdown on breast cancer cell migration was evaluated with the wound healing

\section{A}

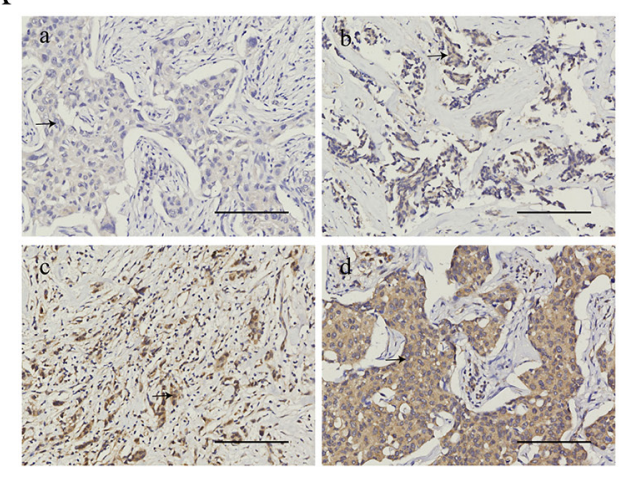

$\mathrm{B}$

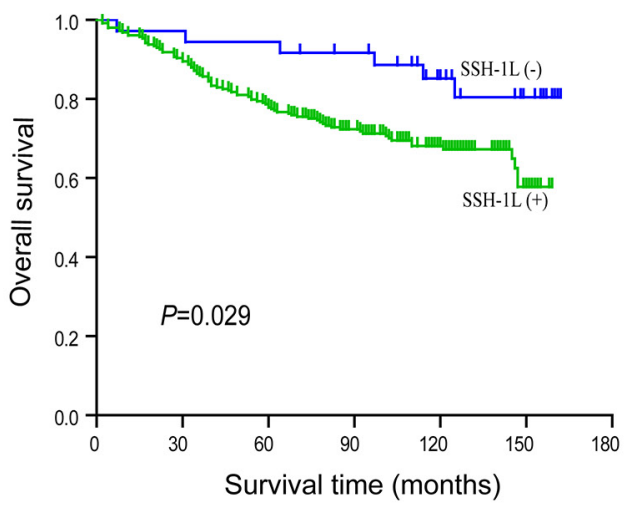

Figure 1: (A) SSH-1L expression in human breast cancer, illustrating representative intensity scores as 0(a); 1(b); 2(c); 3(d). All images were captured at the same magnification, arrow depict tumor cells, bar, $100 \mu \mathrm{m}$. (B) Correlation of SSH-1L expression and survival in all patients, $\mathrm{P}<0.05$ is considered significant. 
Table 1: Kaplan-Meier survival analysis of SSH-1L expression and other clinical pathologic parameters

\begin{tabular}{|c|c|c|c|c|}
\hline \multirow[t]{2}{*}{ Variable } & \multicolumn{3}{|c|}{ Censored } & \multirow[t]{2}{*}{$\mathbf{P}$} \\
\hline & Total N & Survival & Ratio & \\
\hline Age 1 & & & & 0.778 \\
\hline$<35$ & 10 & 7 & $70.00 \%$ & \\
\hline$\geq 35$ & 285 & 200 & $70.18 \%$ & \\
\hline Age 2 & & & & 0.936 \\
\hline$<50$ & 104 & 73 & $70.19 \%$ & \\
\hline$\geq 50$ & 191 & 134 & $70.16 \%$ & \\
\hline Pathology grade & & & & 0.812 \\
\hline 1 & 41 & 27 & $65.85 \%$ & \\
\hline 2 & 228 & 162 & $71.05 \%$ & \\
\hline 3 & 26 & 18 & $69.23 \%$ & \\
\hline T stage & & & & 0.004 \\
\hline 1 & 74 & 58 & $78.38 \%$ & \\
\hline 2 & 188 & 132 & $70.21 \%$ & \\
\hline 3 & 33 & 17 & $51.52 \%$ & \\
\hline $\mathrm{N}$ stage & & & & 0.004 \\
\hline 0 & 134 & 103 & $76.87 \%$ & \\
\hline 1 & 82 & 60 & $73.17 \%$ & \\
\hline 2 & 58 & 33 & $56.90 \%$ & \\
\hline 3 & 21 & 11 & $52.38 \%$ & \\
\hline TNM stage & & & & 0.001 \\
\hline 0 & 1 & 1 & $100.00 \%$ & \\
\hline 1 & 38 & 31 & $81.58 \%$ & \\
\hline 2 & 168 & 127 & $75.60 \%$ & \\
\hline 3 & 88 & 48 & $54.55 \%$ & \\
\hline SSH-1L & & & & 0.029 \\
\hline- & 35 & 29 & $82.86 \%$ & \\
\hline+ & 259 & 177 & $68.34 \%$ & \\
\hline Her-2 & & & & 0.588 \\
\hline- & 226 & 159 & $70.35 \%$ & \\
\hline+ & 69 & 48 & $69.57 \%$ & \\
\hline
\end{tabular}

assay. After $24 \mathrm{~h}$, loss of SSH-1L reduced the migration of SK-BR-3 and MDA-MB-231 cells relative to control cells (Figure 3A-3D). However, cell proliferation was unaltered by SSH-1L deficiency, indicating that the observed effect was not due to growth inhibition (Figure 3E, 3F). Thus, SSH-1L is specifically involved in breast cancer cell migration and may thereby regulate cell invasion and metastasis.

\section{Insulin activates SSH-1L/cofilin signaling in a PI3K-dependent manner}

Insulin induces the activation of LIMK1 and consequently of cofilin to regulate cell membrane protrusion. PI3K plays a critical role in cofilin dephosphorylation via SSH-1L activation [13]. We therefore investigated the role of insulin and the PI3K-dependence of 
Table 2: Multivariate Cox regression analysis of prognostic factors

\begin{tabular}{lcccccc}
\hline & B & Exp(B) & SE & P & \multicolumn{2}{c}{$\mathbf{9 5 \% C I ~}$} \\
\cline { 5 - 7 } & & & & & Lower & Upper \\
\hline Age & 0.184 & 1.202 & 0.23 & 0.422 & 0.766 & 1.866 \\
T stage & 0.405 & 1.499 & 0.226 & 0.073 & 0.963 & 2.332 \\
N stage & 0.144 & 1.155 & 0.196 & 0.464 & 0.786 & 1.696 \\
TNM stage & 0.306 & 1.358 & 0.357 & 0.391 & 0.675 & 2.732 \\
SSH-1L & 0.889 & 2.434 & 0.428 & 0.038 & 1.052 & 5.633 \\
\hline
\end{tabular}

Table 3: Correlation of SSH-1L expression and lymph node metastasis of breast cancer

\begin{tabular}{lcccc}
\hline Variable & \multicolumn{3}{c}{ Number of patients } & P \\
\cline { 2 - 4 } & Total & Metastasis & Ratio & 0.017 \\
\hline SSHIL & & & & \\
- & 36 & 14 & $38.89 \%$ & \\
+ & 259 & 146 & $56.37 \%$ & \\
\hline
\end{tabular}

SSH-1L/cofilin signaling in breast cancer cells and found that insulin treatment induced cofilin dephosphorylation without altering SSH-1L expression (Figure 4A, 4B). Cofilin phosphorylation was increased upon treatment with wortmannin or SSH-1L knockdown in the presence or absence of insulin (Figure 4A). These results demonstrate that insulin induces cofilin dephosphorylation by enhancing SSH-1L activity in a PI3K-dependent manner.

A

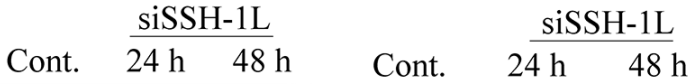

SSH-1L

$\beta$-actin
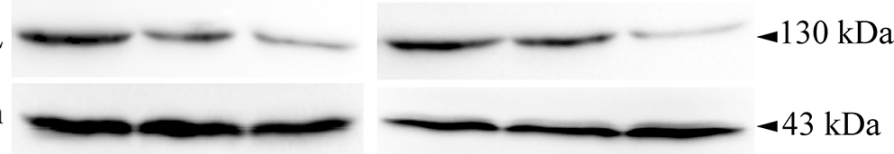

MDA-MB-231

SK-BR-3
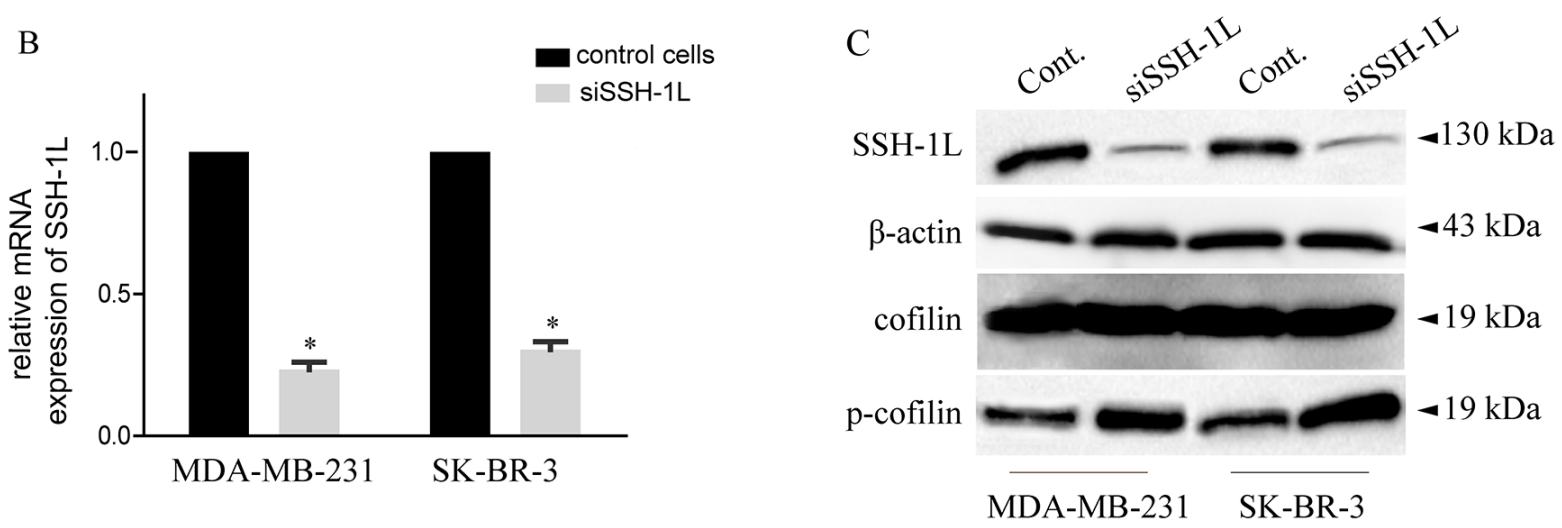

Figure 2: SSH-1L expression was detected in human breast cancer cell lines and loss of SSH-1L decrease the expression of cofilin and increase expression of p-cofilin. (A) SSH-1L expression in different cell lines detected by western-blot, each cell line grouped by control, transfected with siSSH-1L after $24 \mathrm{~h}$ and $48 \mathrm{~h}$. (B) Relative mRNA expression after knockdown of SSH-1L for $48 \mathrm{~h}$ in MDA-MB-231 and SK-BR-3 cells analyzed by RT-PCR. * $\mathrm{P}<0.05$. (C) The expression of SSH-1L, cofilin, p-cofilin, were detected in MDA-MB-231 and SK-BR-3 cells after knockdown of SSH-1L for 48h. The expression of actin was used to normalize the loading volume. 
A

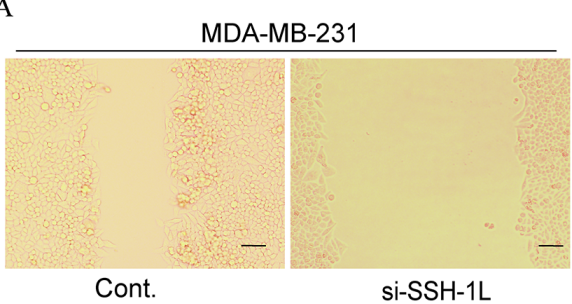

$\mathrm{C}$

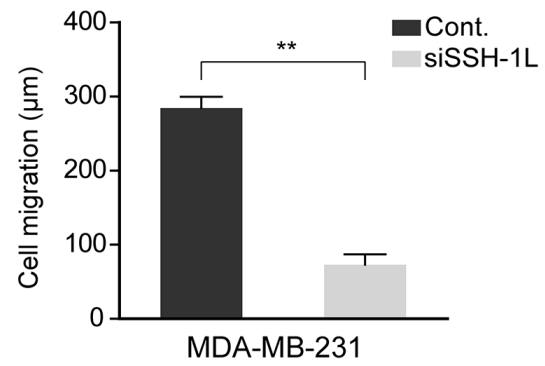

E

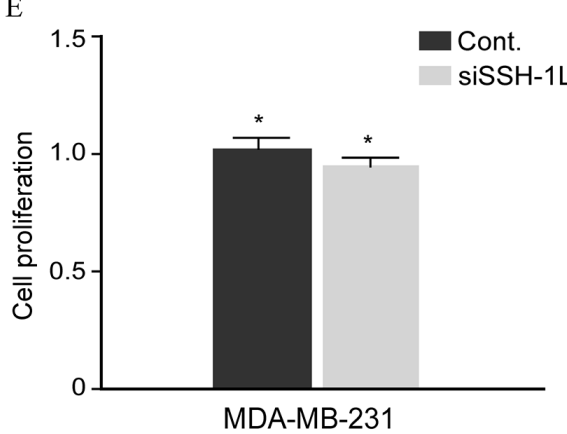

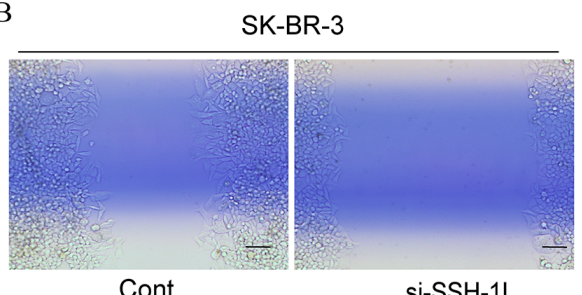

$\mathrm{D}$

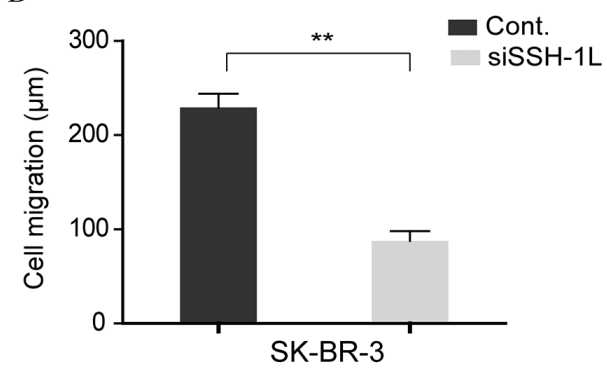

$\mathrm{F}$

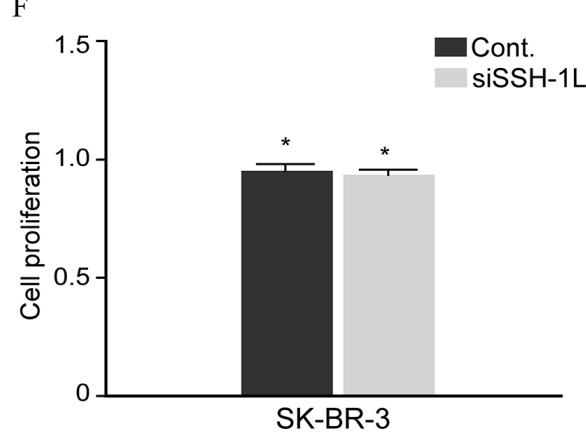

Figure 3: Inhibition of SSH-1L expression reduces breast cancer cell migration. (A) and (B) Cell migration of MDA-MB-231 and SK-BR-3 were assessed by cell scratching assay. (C) and (D) Qualification of cell migration was analyzed by image J and data was performed by t-test, **, P<0.01. (E) and (F) Relative cell proliferation was estimated by MTT after SSH-1L was knocked down for $72 \mathrm{~h}$, $*, \mathrm{P}<0.5$.

A

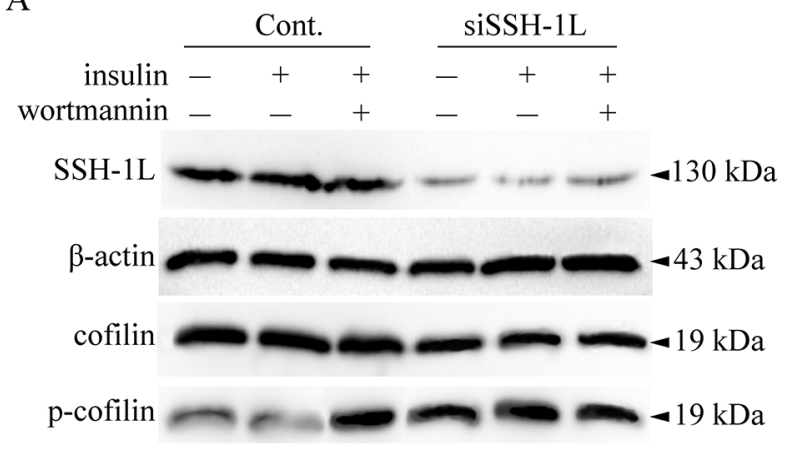

MCF-7
B

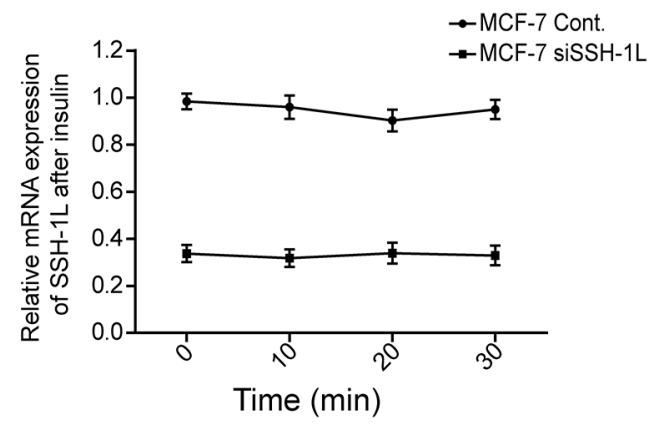

Figure 4: Insulin induce SSH-1L activation and cofilin dephosphorylation without altering the expression of SSH-1L in a PI3K-dependent manner. (A) The expression and siSSH-1L groups were incubated with or without $10 \mu \mathrm{g} / \mathrm{ml}$ insulin for $30 \mathrm{minutes}$, then the insulin $(+)$ cells of each group were incubated with or without wortmannin, a PI3K inhibitor. (B) The relative mRNA expression of SSH-1L was detected by RT-PCR respectively at $0,10,20,30$ minutes after incubation of MCF-7 cell with insulin. 


\section{DISCUSSION}

In this study, we showed that SSH-1L was overexpressed in $88.1 \%$ of clinical breast cancer specimens, and that this predicted breast cancer lymph node metastasis and poor survival in patients. Loss of SSH-1L increased cofilin phosphorylation and inhibited cell migration in SK-BR-3 and MDA-MB-231 human breast cancer cell lines, indicating that SSH-1L regulates cell motility via cofilin dephosphorylation. In insulin-stimulated MCF-7 cells, increasedSSH$1 \mathrm{~L}$ activation reduced cofilin phosphorylation, while SSH-1L knockdown abolished insulin-induced cofilin dephosphorylation. SSH-1L is a phosphatase that specifically dephosphorylates cofilin at Ser-3 [11] and thus plays a critical role in actin dynamics, which in turn modulate tumor metastasis and invasion [25]. The results presented here demonstrate for the first time that SSH$1 \mathrm{~L}$ modulates breast cancer metastasis via regulation of cofilin signaling.

Cofilin is a member of the actin-depolymerizing factor/cofilin family of proteins $[6,26]$ that regulate the formation of the actin cytoskeleton [22]. Tumor invasion and metastasis - which is the main cause of cancer-related death-is directly associated with cofilin activity [23, 27]. Our previous work also showed that the phosphorylation status of cofilin is associated with survival in breast cancer patients [28]. Another study reported that the overall activation of the cofilin pathway determines the invasive and metastatic phenotype of tumor cells [29].

As a regulator of cofilin, $\mathrm{SSH}-1 \mathrm{~L}$ affects tumor migration by altering the activity of cofilin/F-actin. The cofilin-phosphatase activity of SSH-1L is increased by binding to F-actin [30-32] via Trp-458 at the C-terminus of the phosphatase domain and the N-terminal LHK and C-terminal LKR motifs. A pleckstrin homology-like domain at the N-terminus of SSH-1L was also shown to be involved in F-actin binding and cofilin phosphatase activity [33].

In conclusion, our results reveal an important role for SSH-1L/cofilin signaling in breast cancer. These findings provide a basis for the development of drugs that target SSH-1L to prevent breast cancer progression and improve patient outcome.

\section{MATERIALS AND METHODS}

\section{Tissue specimens and patients' clinical information}

The study population included 295 invasive breast cancer patients who underwent surgery as initial treatment between 2001 and 2008 at hospitals in Shanghai, Jiangsu, and Zhejiang. Patients provided written, informed consent for participation in the study at the National Engineering Center for Biochip at Shanghai. A diagnosis of breast cancer was confirmed based on postoperative pathological findings and followed standard guidelines among all these hospitals during the period. The following clinical characteristics were recorded for each patient: age; gender; tumor size; lymph node invasion; tumor-node-metastasis (TNM) stage; and estrogen and progesterone receptor and human epidermal growth factor receptor (HER)-2 status. Patients with breast cancer were followed from the date of surgery to the date of death or last follow-up.

\section{Immunohistochemistry}

SSH-1L, cofilin, and Aur-A expression in clinical specimens was detected by immunohistochemistry using anti-SSH-1L (ab76943, 1:100) and -cofilin-1 (ab42824, 1:1500) antibodies (both from Abcam, Cambridge, MA, USA) and anti-Aur-A antibody (\#14475, 1:50; Cell Signaling Technology, Danvers, MA, USA). The degree of immunoreactivity in the cytoplasm was evaluated by two experienced pathologists blinded to patients' clinical information. Expression was graded as positive (high staining intensity, $>5 \%$ ) or negative (low staining intensity, 0-5\%) [24]. The slides were scanned using a ScanScope scanner (Aperio, Vista, CA, USA), and images of representative areas were acquired using Image Scope software (Leica, Wetzlar, Germany).

\section{Cell culture}

MDA-MB-231, MCF-7, and SK-BR-3 human breast cancer cell lines were purchased from the American Type Culture Collection (Manassas, VA, USA). MDA-MB-231 cells were maintained in L-15 medium (Gibco, Grand Island, NY, USA) supplemented with $10 \%$ fetal bovine serum (FBS; Gibco) at $37^{\circ} \mathrm{C}$ in a humidified incubator. MCF-7 cells were maintained in Dulbecco's Modified Eagle's medium (Gibco) supplemented with 10\% FBS and 10mM estrogen (E2758; Sigma-Aldrich, St. Louis, MO, USA). SK-BR-3 cells were cultured in MyCoy'5a medium (Gibco) supplemented with $10 \% \mathrm{FBS}$ at $37^{\circ} \mathrm{C}$ in a humidified atmosphere of $5 \% \mathrm{CO}_{2}-$ $5 \%$ air. Insulin was purchased from Sigma-Aldrich and wortmannin was obtained from Selleck Chemicals (S2578; Houston, TX, USA).

\section{Antibodies and quantitative real-time (qRT-) PCR primers}

Antibodies specific for SSH-1L and cofilin-1 were purchased from Abcam (ab76943 and ab42824, respectively). Antibodies against Aur-A and phospho-cofilin-1 were from Cell Signaling Technology (\#14475 and \#3313, respectively; Danvers, MA, USA). Antibody against $\beta$-actin was purchased from Santa Cruz Biotechnology (sc-81178; Santa Cruz, CA, USA). Horseradish peroxidase(HRP)conjugated anti-mouse and -rabbit antibodies were from Cell Signaling Technology (\#7076 and\#7078, respectively). The following sense and anti-sense primers were used for qRT- 
PCR: SSH-1L, 5'-GGAAGAATCGTCACCCAA-3' and 5'-CAGGCGGTAGAAGAAAGG-3'; and $\beta$-actin, 5'-GT GGACATCCGCAAAGAC-3' and 5'-TGGGTGCCAGGG CAGTGATC-3'.

\section{Short interfering (si)RNA-mediated knockdown of SSH-1L gene expression}

An siRNA oligonucleotide (5'-ucgucacccaagaaa gauatt-3') was used to knock down $S S H-1 L$ expression, with lacZ-specific siRNA used as a negative control. Breast cancer cells were seeded in a 6 -well flat-bottom plate $\left(2 \times 10^{5}\right.$ per well). At $70 \%$ confluence, the culture medium was replaced and 30 min later, cells were transfected with SSH-1L or control siRNA using Lipofectamine 2000 (Invitrogen, Carlsbad, CA, USA) according to the manufacturer's protocol.

\section{Western blotting}

Cells were seeded in a 6-well plate until the number reached $1 \times 10^{6}$ per well. The supernatant was discarded and cells were washed with phosphatebuffered saline before homogenization on ice for $30 \mathrm{~min}$ in radio immuno-precipitation assay buffer containing $1 \%$ phenylmethylsulfonyl fluoride. Cells were collected by scraping into sterile tube and centrifuged at $12,000 \times \mathrm{g}$ for 15 min at $4^{\circ} \mathrm{C}$. The supernatant was collected and protein concentration was determined with the bicinchoninic acid assay (Thermo Fisher Scientific, Waltham, MA, USA). Equal amounts of protein were resolved by sodium dodecyl sulfatepolyacrylamide gel electrophoresis on a MiniPROTEAN Tetra Cell (Bio-Rad, Hercules, CA, USA) and transferred to a $0.45-\mu \mathrm{m}$ polyvinylidene difluoride membrane (Millipore, Billerica, MA, USA) that was blocked overnight with 5\% skimmed milk diluted in Tris-buffed saline with0.1\% Tween-20 (TBST) for $1 \mathrm{~h}$ followed by overnight incubation at $4^{\circ} \mathrm{C}$ with the primary antibodies. The membrane was washed three times with TBST and then incubated with HRPconjugated secondary antibody for $1 \mathrm{~h}$ at room temperature. Protein bands were visualized using a chemiluminescence reagent (Goodbio Biotechnology, Wuhan, China). The blots were imaged with a Molecular Imager ChemiDoc XRSt (Bio-Rad) and ImageLab v.4.1 software (Bio-Rad) was used to determine the background-subtracted density of the bands.

\section{Cell scratching assay}

Cells were seeded in a 6-well flat-bottom plate at $2 \times 10^{5}$. Twenty-four hours later, cells were transfected with SSH-1L or control siRNA. Forty-eight hours later, cells were serum starved with $0.1 \%$ FBS in EBM medium for $24 \mathrm{~h}$. A scratch was made to the bottom of the well through the cells with a sterile $200 \mu$ pipette tip. After washes to remove those that were non-adherent, the cells were cultured in EBM medium containing 0.1\% FBS and examined at 0, 16 and 24h with a BZ-9000 epifluorescence microscope (Keyence, Itasca, IL, USA). Quantification was performed using ImageJ software (National Institutes of Health, Bethesda, MD, USA).

\section{Proliferation assay}

Cells were seeded in 96-well plates at $2 \times 10^{3}$ cells/ well. Twenty-four hours later, cells were transfected with SSH-1L or control siRNA using Lipofectamine 2000 (Invitrogen, Carlsbad, CA, USA) according to the manufacturer's protocol. Forty-eight hours later, cells were serum starved with $0.1 \%$ FBS in EBM medium for $24 \mathrm{~h}$; A proliferation assay was carried out with cell counting kit-8 (Sigma-Aldrich, St. Louis, MO, USA)following the protocol provided by the producer. Data are expressed as the mean $\pm \mathrm{SD}$ of quadruplicate values.

\section{Statistical analysis}

Group means were compared with the Student's t test and Pearson's $\chi^{2}$ test was used to assess correlations between variables. The Cox regression proportional hazards model was used to estimate hazard ratios for lymph node metastasis based on Aur-A and CFL-1 expression. All statistical tests were two-sided and a $\mathrm{P}$ value $<0.05$ was considered statistically significant. Analyses were performed using SPSS v.19 software (SPSS, Chicago, IL. USA).

\section{Author contributions}

Chen Chen: Formulation, validation, investigation, methodology, designing the experiments, writing- initial draft, writing-review and editing. Yusufu Maimaiti: Validation, resources, performing the experiments, data curation, writing- initial draft. Shen Zhijun: Programming, software, methodology, performing the experiment, data curation. Liu Zeming: Verification, formal analysis, methodology. Guo Yawen: Provision of study materials, reagents and materials. Data duration. Yu Pan: Formal analysis, software, investigation, performing the experiments. Huang Tao: Oversight and leadership responsibility for the research activity planning and execution, supervision, project administration, and funding acquisition, responsible for the overall content as guarantor.

\section{ACKNOWLEDGMENTS}

We would like to thank Editage (www.editage.com) for language editing.

\section{CONFLICTS OF INTEREST}

The authors have no conflicts of interest to declare.

\section{FUNDING}

No. 


\section{REFERENCES}

1. Pollard TD, Cooper JA. Actin, a central player in cell shape and movement. Science. 2009; 326: 1208-12. https://doi. org/10.1126/science.1175862.

2. Bugyi B, Carlier MF. Control of actin filament treadmilling in cell motility. Annu Rev Biophys. 2010; 39: 449-70. https://doi.org/10.1146/annurev-biophys-051309-103849.

3. Pollard TD, Borisy GG. Cellular motility driven by assembly and disassembly of actin filaments. Cell. 2003; 112: 453-65. doi: S009286740300120X.

4. Ghosh M, Song X, Mouneimne G, Sidani M, Lawrence DS, Condeelis JS. Cofilin promotes actin polymerization and defines the direction of cell motility. Science. 2004; 304: 743-6. https://doi.org/10.1126/science.1094561.

5. Gungabissoon RA, Bamburg JR. Regulation of growth cone actin dynamics by ADF/cofilin. J Histochem Cytochem. 2003; 51: 411-20.

6. Maciver SK, Hussey PJ. The ADF/cofilin family: actinremodeling proteins. Genome Biol. 2002; 3: reviews3007.

7. Horita Y, Ohashi K, Mukai M, Inoue M, Mizuno K. Suppression of the invasive capacity of rat ascites hepatoma cells by knockdown of Slingshot or LIM kinase. J Biol Chem. 2008; 283: 6013-21. https://doi.org/10.1074/jbc. M706538200.

8. Nishita M, Tomizawa C, Yamamoto M, Horita Y, Ohashi K, Mizuno K. Spatial and temporal regulation of cofilin activity by LIM kinase and Slingshot is critical for directional cell migration. J Cell Biol. 2005; 171: 349-59. https://doi.org/10.1083/jcb.200504029.

9. Huang TY, DerMardirossian C, Bokoch GM. Cofilin phosphatases and regulation of actin dynamics. Curr Opin Cell Biol. 2006; 18: 26-31. https://doi.org/10.1016/j. ceb.2005.11.005.

10. Cai L, Marshall TW, Uetrecht AC, Schafer DA, Bear JE. Coronin 1B coordinates Arp2/3 complex and cofilin activities at the leading edge. Cell. 2007; 128: 915-29. https://doi.org/10.1016/j.cell.2007.01.031.

11. Niwa R, Nagata-Ohashi K, Takeichi M, Mizuno K, Uemura T. Control of actin reorganization by Slingshot, a family of phosphatases that dephosphorylate ADF/cofilin. Cell. 2002; 108: 233-46. doi: S0092867401006389.

12. Kaji N, Ohashi K, Shuin M, Niwa R, Uemura T, Mizuno $\mathrm{K}$. Cell cycle-associated changes in Slingshot phosphatase activity and roles in cytokinesis in animal cells. J Biol Chem. 2003; 278: 33450-5. https://doi.org/10.1074/jbc. M305802200.

13. Nishita M, Wang Y, Tomizawa C, Suzuki A, Niwa R, Uemura T, Mizuno K. Phosphoinositide 3-kinase-mediated activation of cofilin phosphatase Slingshot and its role for insulin-induced membrane protrusion. J Biol Chem. 2004; 279: 7193-8. https://doi.org/10.1074/jbc.M312591200.
14. Wang Z, Wang M, Carr BI. Involvement of receptor tyrosine phosphatase DEP-1 mediated PI3K-cofilin signaling pathway in sorafenib-induced cytoskeletal rearrangement in hepatoma cells. J Cell Physiol. 2010; 224: 559-65. https:// doi.org/10.1002/jcp.22160.

15. Vitolo MI, Boggs AE, Whipple RA, Yoon JR, Thompson K, Matrone MA, Cho EH, Balzer EM, Martin SS. Loss of PTEN induces microtentacles through PI3K-independent activation of cofilin. Oncogene. 2013; 32: 2200-10. https:// doi.org/10.1038/onc.2012.234.

16. Homma Y, Kanno S, Sasaki K, Nishita M, Yasui A, Asano T, Ohashi K, Mizuno K. Insulin receptor substrate-4 binds to Slingshot-1 phosphatase and promotes cofilin dephosphorylation. J Biol Chem. 2014; 289: 26302-13. https://doi.org/10.1074/jbc.M114.565945.

17. Wang LH, Xiang J, Yan M, Zhang Y, Zhao Y, Yue CF, Xu J, Zheng FM, Chen JN, Kang Z, Chen TS, Xing D, Liu Q. The mitotic kinase Aurora-A induces mammary cell migration and breast cancer metastasis by activating the Cofilin-Factin pathway. Cancer Res. 2010; 70: 9118-28. https://doi. org/10.1158/0008-5472.CAN-10-1246.

18. Nicolini A, Giardino R, Carpi A, Ferrari P, Anselmi L, Colosimo S, Conte M, Fini M, Giavaresi G, Berti P, Miccoli P. Metastatic breast cancer: an updating. Biomed Pharmacother. 2006; 60: 548-56. https://doi.org/10.1016/j. biopha.2006.07.086.

19. Chen W, Zheng R, Zhang S, Zhao P, Li G, Wu L, He J. Report of incidence and mortality in China cancer registries, 2009. Chin J Cancer Res. 2013; 25: 10-21. https://doi. org/10.3978/j.issn.1000-9604.2012.12.04.

20. Li T, Mello-Thoms C, Brennan PC. Descriptive epidemiology of breast cancer in China: incidence, mortality, survival and prevalence. Breast Cancer Res Treat. 2016; 159: 395-406. https://doi.org/10.1007/ s10549-016-3947-0.

21. Li Z, Kang Y. Emerging therapeutic targets in metastatic progression: a focus on breast cancer. Pharmacol Ther. 2016; 161: 79-96. https://doi.org/10.1016/j.pharmthera.2016.03.003.

22. Wang W, Eddy R, Condeelis J. The cofilin pathway in breast cancer invasion and metastasis. Nat Rev Cancer. 2007; 7: 429-40. https://doi.org/10.1038/nrc2148.

23. Wang W, Mouneimne G, Sidani M, Wyckoff J, Chen X, Makris A, Goswami S, Bresnick AR, Condeelis JS. The activity status of cofilin is directly related to invasion, intravasation, and metastasis of mammary tumors. J Cell Biol. 2006; 173: 395404. https://doi.org/10.1083/jcb.200510115.

24. Maimaiti Y, Jie T, Jing Z, Changwen W, Pan Y, Chen C, Tao H. Aurora kinase A induces papillary thyroid cancer lymph node metastasis by promoting cofilin-1 activity. Biochem Biophys Res Commun. 2016; 473: 212-8. https:// doi.org/10.1016/j.bbrc.2016.03.081.

25. Kanellos G, Frame MC. Cellular functions of the ADF/ cofilin family at a glance. J Cell Sci. 2016; 129: 3211-8. https://doi.org/10.1242/jcs.187849. 
26. Bamburg JR. Proteins of the ADF/cofilin family: essential regulators of actin dynamics. Annu Rev Cell Dev Biol. 1999; 15: 185-230. https://doi.org/10.1146/annurev. cellbio.15.1.185.

27. Sidani M, Wessels D, Mouneimne G, Ghosh M, Goswami S, Sarmiento C, Wang W, Kuhl S, El-Sibai M, Backer JM, Eddy R, Soll D, Condeelis J. Cofilin determines the migration behavior and turning frequency of metastatic cancer cells. J Cell Biol. 2007; 179: 777-91. https://doi. org/10.1083/jcb.200707009.

28. Maimaiti Y, Liu Z, Tan J, Abudureyimu K, Huang B, Liu C, Guo Y, Wang C, Nie X, Zhou J, Huang T. Dephosphorylated cofilin expression is associated with poor prognosis in cases of human breast cancer: a tissue microarray analysis. Onco Targets Ther. 2016; 9: 6461-6. https://doi.org/10.2147/OTT. S107321.

29. Tsai CH, Chiu SJ, Liu CC, Sheu TJ, Hsieh CH, Keng PC, Lee YJ. Regulated expression of cofilin and the consequent regulation of $\mathrm{p} 27$ (kip1) are essential for $\mathrm{G}(1)$ phase progression. Cell Cycle. 2009; 8: 2365-74. https://doi. org/10.4161/cc.8.15.9072.

30. Mizuno K. Signaling mechanisms and functional roles of cofilin phosphorylation and dephosphorylation. Cell Signal. 2013; 25: 457-69. https://doi.org/10.1016/j. cellsig.2012.11.001.
31. Yamamoto M, Nagata-Ohashi K, Ohta Y, Ohashi K, Mizuno $\mathrm{K}$. Identification of multiple actin-binding sites in cofilinphosphatase Slingshot-1L. FEBS Lett. 2006; 580: 1789-94. https://doi.org/10.1016/j.febslet.2006.02.034.

32. Kurita S, Watanabe Y, Gunji E, Ohashi K, Mizuno K. Molecular dissection of the mechanisms of substrate recognition and F-actin-mediated activation of cofilinphosphatase Slingshot-1. J Biol Chem. 2008; 283: 3254252. https://doi.org/10.1074/jbc.M804627200.

33. Takahashi K, Okabe H, Kanno SI, Nagai T, Mizuno K. A pleckstrin homology-like domain is critical for F-actin binding and cofilin-phosphatase activity of Slingshot-1. Biochem Biophys Res Commun. 2016; 482: 686-92. https:// doi.org/10.1016/j.bbrc.2016.11.095. 